\title{
An incidental finding of a subcutaneous retained firearm pellet mistaken for a retained capsule endoscopy identified by colonoscopy with fluoroscopy and radiological imaging
}

\author{
Naser Mubarak ${ }^{*}$, Andrew Collins ${ }^{1}$, Hadi Hemaidan², Sami Hemaidan² and Ammar Hemaidan ${ }^{3}$ \\ ${ }^{1}$ University of Central Florida College of Medicine, Orlando, FL, USA \\ ${ }^{2}$ University of Florida, Gainesville, FL, USA \\ ${ }^{3}$ Florida State University College of Medicine, Tallahassee, FL, USA
}

\begin{abstract}
Capsule endoscopy retention is a rare event that occurs in $1.5-2.4 \%$ of cases utilizing the imaging method. Patients with known Crohn's disease and obstructive tumours carry an increased risk of occurrence with up to a $13 \%$ risk of retention. Findings of foreign bodies during examinations or medical procedures are often unexpected and an unlikely occurrence. In this case, we report a 79-year-old male who underwent an interventional colonoscopy after initial capsule endoscopy retention. During the colonoscopy, a subcutaneous abdominal foreign body, which was later identified to be an impacted ball bearing gun pellet, was observed under fluoroscopy.
\end{abstract}

\section{Introduction}

The use of capsule endoscopy (CE) has improved and facilitated our ability to observe and diagnose potential complications within the small bowel mucosa. Since its inception, the use of CE has been routinely used in cases of obscure gastrointestinal bleeding and in the evaluation of patients with known and suspected Crohn's disease [1,2]. Previous studies have demonstrated the superiority of detecting small bowel lesions with capsule endoscopy compared to other diagnostic methods [2-5]. One of the greater risks and considered complications of capsule endoscopy is capsule retention, which is defined as the presence of the capsule endoscope within the digestive tract for a minimum of 2-weeks. Risk of indefinite CE retention in the small bowel can also occur unless a targeted medical or surgical intervention is initiated [6-9]. We present an interesting case of a subcutaneous abdominal foreign body found during an interventional colonoscopy in response to capsule endoscopy retention.

\section{Case presentation}

A 79-year-old male presented to the emergency department after two days experiencing melena. The patient had three episodes of black stools and a further two episodes of dark bloody stools with clots present, but no change is bowel habits. He had no history of aspirin, NSAID, or blood thinner use, and had no history of alcohol use or cirrhosis. The patient also denied vomiting or abdominal pain and had negative familial history for colon cancer. The patient stated that he had gastric ulcers approximately 7-10 years prior but had not had incidents since. Initial lab reports revealed a hemoglobin of $4.8 \mathrm{~g} / \mathrm{dL}$, BUN of $20 \mathrm{mEq} / \mathrm{L}$, and creatinine of $1.2 \mathrm{mEq} / \mathrm{L}$, all consistent with upper gastrointestinal bleeding. The patient was treated with two units of packed red blood cells and the following labs showed a rebound of hemoglobin levels to $7.3 \mathrm{~g} / \mathrm{dL}$. A computerized tomography (CT) scan of the abdomen and pelvis displayed diverticulosis and an adrenal mass. A follow-up blood smear showed normocytic anemia and the patient tested positive for a hemoccult test but presented with a lack of active bleeding. A diagnostic colonoscopy showed severe diverticulosis throughout the entire examined colon, internal hemorrhoids, a normal mucosal surface, and no active bleeding.

\section{Imaging features}

Multiple contiguous axial images were obtained through the abdomen and pelvis following bolus infusion of $96 \mathrm{~mL}$ Omnipaque 350 non-ionic water-soluble contrast. The imaging showed cholelithiasis, diverticulosis without diverticulitis, atherosclerosis, right adrenal mass $(4.0 \times 2.9 \mathrm{~cm}$ in anteroposterior and transverse respectively), prominent prostate, a left renal cyst $(1.6 \mathrm{~cm})$, and no evidence of bowel obstruction.

Following this imaging, the patient was given a pill-camera CE, however, the patient never retrieved the capsule. Imaging was once again attempted using another CE; however, the pill-capsule could not be located for a second time, and a kidney-ureter-bladder $\mathrm{x}$-ray was obtained to locate it. A colonoscopy and rectal exam were performed under video guidance to the cecum, finding colon diverticular disease prominent in the descending colon and a small polyp $(3 \times 4 \mathrm{~mm})$ in the transverse colon removed for biopsy, but the pill-capsule endoscopy was still unable to be located. A fluoroscopy machine was used to scan the abdomen, showing a $6 \times 3 \mathrm{~mm}$ rectangular foreign body in

*Correspondence to: Naser Mubarak, University of Central Florida College of Medicine, USA, Tel: (386) 898-3748, E-mail: Nmubarak94@knights.ucf.edu

Key words: capsule endoscopy, capsule retention, diverticulosis

Received: November 02, 2020; Accepted: November 16, 2020; Published: November 19, 2020 
Mubarak N (2020) An incidental finding of a subcutaneous retained firearm pellet mistaken for a retained capsule endoscopy identified by colonoscopy with fluoroscopy and radiological imaging

the sigmoid area overlying the left iliac crest, potentially the entrapped capsule in a diverticulum. However, it was not correlating with the colonoscopy scope position, and on further examination seemed to be a foreign body under the skin. Since the scans did not show the pill-capsule, it is likely that the patient passed the capsules in his stool without his knowledge. However, this foreign body under the patient's skin was an interesting finding, and revealed the patient's history of gunshot wound, as the object was identified as a ball bearing (BB) gun pellet lodged in the patient's abdominal skin.

\section{Discussion}

The risk of capsule endoscopy retention ranges from $1.4-2.5 \%$ in the general population and increases to $13 \%$ in patients with Crohn's disease, obstructive tumors, and diaphragm disease, often due to side effects of non-steroidal anti-inflammatory pharmaceuticals $[9,10]$. Patients with obscure gastrointestinal bleeding are at lowest risk of capsule retention.

Foreign bodies are considered a significant cause of morbidity in soft tissue injuries and often unexpectedly present themselves to clinicians as localized inflammation and cellulitis, abscess formation, and delayed wound healing. Removal of neglected soft tissue foreign bodies may be complex due to indurations, fibrotic scarring, and granulated tissue [11]. Additionally, the composition of the foreign body will also influence evaluation and removal. Metal objects in soft tissue pose a lower risk of infection than organic matter. Inert metal foreign bodies may not have to be removed, because removal may induce more trauma than simply leaving them in place. The decision to remove a foreign body is also based on symptoms or risk of complications.

This case presented with capsule retention twice before an interventional colonoscopy was scheduled and the BB-gun pellet was found through proxy of fluoroscopy when the scope location did not correlate with the region of the foreign body. It is possible the $\mathrm{CE}$ became trapped in a diverticulum, as most cases of retained CE are asymptomatic, or more likely that the patient passed them through unknowingly. Very few cases of symptomatic bowel obstruction requiring surgical or endoscopic intervention of the impacted capsule have been reported $[12,13]$.

\section{Conclusion}

A 79-year-old male presented to the emergency department after episodes of black and dark bloody stools. A CT of the abdomen and pelvis displayed diverticulosis and an adrenal mass. Follow-up blood smears showed a normocytic anemia and the patient tested positive for hemoccult but showed no signs of active bleeding. A diagnostic colonoscopy demonstrated severe diverticulosis throughout the entire examined colon, internal hemorrhoids, a normal mucosal surface, and verified the lack of active bleeding. Capsule endoscopy was performed twice without retrieval followed by an interventional colonoscopy, in which a subcutaneous abdominal foreign body was identified via fluoroscopy. Follow-up imaging later identified the object as a BB-gun pellet from a previous gunshot wound encountered by the patient.

\section{References}

1. Triester SL, Leighton JA, Fleischer DE, Hara AK, Heigh R, et al. (2004) Yield of Capsule Endoscopy (Ce) Compared to Other Modalities in Patients with Obscure GI Bleeding (OGIB). Am J Gastroenterol 99: S308.

2. Kornbluth JF, Colombel JA, Leighton, Loftus E (2005) ICCE Consensus for Inflammatory Bowel Disease. Endoscopy 37: 1051-1054.

3. Marmo R, Rotondano G, Piscopo R, Bianco MA, Cipolletta L (2005) Meta-analysis: capsule enteroscopy vs. conventional modalities in diagnosis of small bowel diseases. Aliment Pharm Ther 22: 595-604.

4. Legnani P, Kornbluth A (2005) Video capsule endoscopy in inflammatory bowel disease. Curr Opin Gastroenterol 21: 438-442. [Crossref]

5. Liao Z, Gao R, Xu C, Li ZJ (2010) Indications and detection, completion, and retention rates of small-bowel capsule endoscopy: a systematic review. Gastrointest Endosc 71: 280-286. [Crossref]

6. Postgate A, Tekkis P, Patterson N, Fitzpatrick A, Bassett P, et al. (2009) Are bowe purgatives and prokinetics useful for small-bowel capsule endoscopy? A prospective randomized controlled study. Gastrointest Endosc 69: 1120-1128. [Crossref]

7. Villa F, Signorelli C, Rondonotti E, Franchis R (2006) Preparations and prokinetics. Gastrointest Endosc Clin N Am 16: 211-220. [Crossref]

8. Cheon JH, Kim YS, Lee IS (2007) Can we predict spontaneous capsule passage after retention? A nationwide study to evaluate the incidence and clinical outcomes of capsule retention. Endoscopy 39: 1046-1052. [Crossref]

9. Cheifetz AS, Kornbluth A, Legnani P (2006) The risk of retention of the capsule endoscope in patients with known or suspected Crohn's disease. Am J Gastroenterol 101: 2218-2222. [Crossref]

10. Li F, Gurudu SR, Petris G (2008) Retention of the capsule endoscope: a single-center experience of 1000 capsule endoscopy procedures. Gastrointest Endosc 68: 174-180. [Crossref]

11. Halaas GW (2007) Management of foreign bodies in the skin. Am Fam Physician 76 683-688.

12. Boysen M, Ritter M (2010) Small bowel obstruction from capsule endoscopy. West $J$ Emerg Med 11: 71-73. [Crossref]

13. Rogers AM, Kuperman E, Puleo FJ, Shope TR (2008) Intestinal obstruction by capsule endoscopy in a patient with radiation enteritis. JSLS 12: 85-87. [Crossref]

Copyright: (C2020 Mubarak N. This is an open-access article distributed under the terms of the Creative Commons Attribution License, which permits unrestricted use, distribution, and reproduction in any medium, provided the original author and source are credited. 